\title{
Supplement: Temperatures in transient climates: improved methods for simulations with evolving temporal covariances
}

\author{
Andrew Poppick ${ }^{1}$, David J. McInerney ${ }^{2}$, Elisabeth J. Moyer ${ }^{3}$, and Michael L. Stein ${ }^{1}$ \\ ${ }^{1}$ Department of Statistics, University of Chicago \\ ${ }^{2}$ School of Civil, Environmental and Mining Engineering, University of Adelaide \\ ${ }^{3}$ Department of the Geophysical Sciences, University of Chicago
}

\section{S1 Estimating changes in regional and local mean tempera- ture}

The model for changes in covariance, (6), requires an estimate of the changes in regional mean temperature, $\bar{\Delta}_{S}^{(s, B)}(t)$, for scenario $s$. We also need changes in local mean temperature to compute the simulation (4). We estimate these using a modification of the mean emulator described in Castruccio et al. (2014). Write $\bar{\mu}_{S}^{(s)}(t)$ for the regional mean temperature at time $t$ under scenario $s$ in region $S$. We assume that

$$
\begin{aligned}
\bar{\mu}_{S}^{(s)}(t)=\quad \beta_{0, S} & +\beta_{1, S} C^{(s)}(t)+\sum_{k=1}^{K}\left\{\gamma_{k, S} \cos \left(\frac{2 \pi t k}{365}\right)+\zeta_{k, S} \sin \left(\frac{2 \pi t k}{365}\right)\right\} \\
& +\sum_{k=1}^{K}\left\{\gamma_{k, S}^{\prime} C^{(s)}(t) \cos \left(\frac{2 \pi t k}{365}\right)+\zeta_{k, S}^{\prime} C^{(s)}(t) \sin \left(\frac{2 \pi t k}{365}\right)\right\}
\end{aligned}
$$

where

$$
C^{(s)}(t)=\sum_{m=0}^{\infty}\left(1-\phi_{S}\right) \phi_{S}^{m} \log \left(\frac{\left[\mathrm{CO}_{2}\right]^{(s)}(t-m)}{\left[\mathrm{CO}_{2}\right]^{(\mathrm{B})}}\right)
$$

for $\phi_{S} \in[0,1)$, and $\left[\mathrm{CO}_{2}\right]^{(s)}(t)$ and $\left[\mathrm{CO}_{2}\right]^{(\mathrm{B})}$ are the $\mathrm{CO}_{2}$ concentrations under scenario $s$ at time $t$ and under preindustrial conditions, respectively. For the harmonic terms, we take $K=6$. This mean emulator differs from that described in Castruccio et al. (2014) in essentially two ways. First, here we exclude one effect in theirs that was meant to distinguish short term and long term effects of changes in $\left[\mathrm{CO}_{2}\right]$. For smooth, monotonic scenarios like those in our ensemble, it is difficult to distinguish these two effects. Second, whereas their paper was concerned with emulating annual average temperatures, here we are interested in daily temperatures, so we need terms that account for the (possibly changing) mean seasonal cycle; note that the GCM runs use a year of exactly 365 days, hence our representation of the seasonal cycle in terms of harmonics of $1 / 365$. In most regions, any changes to the mean seasonal cycle are small besides an overall increase in mean, although in regions with strong seasonal cycles, the mean seasonal cycle tends to be damped more in winter months than summer months (Figure S1). 

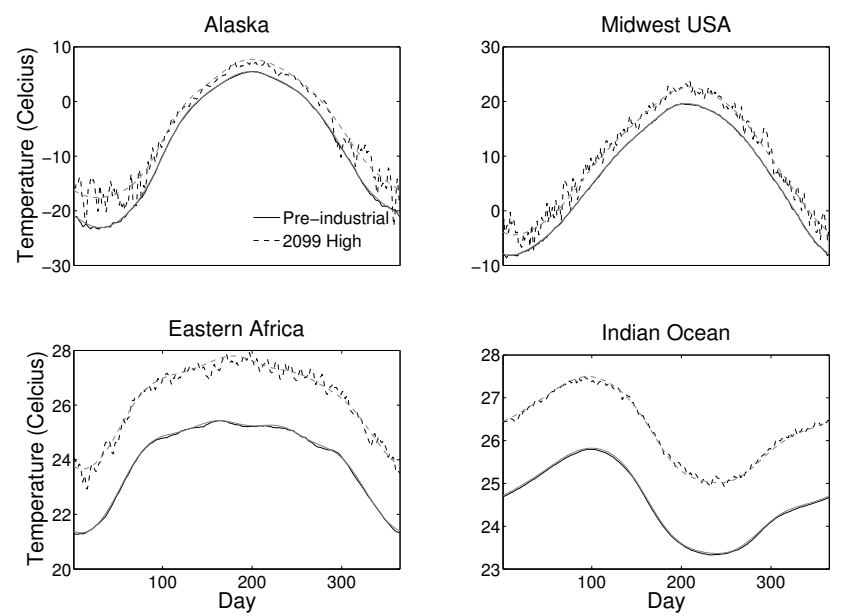

Figure S1: Examples of emulated (gray) and empirical (black) mean seasonal cycles in four regions compared between the preindustrial climate (solid) and the climate under the high scenario in the year 2099 (dashed). The emulations are fitted according to (S1). The empirical estimates are obtained by averaging across grid cells in the region of interest and across time (for the equilibrated preindustrial run) or across realizations (for the high scenario realizations). The empirical estimates of the 2099 seasonal cycles (red) are noisy because they represent averages across only eight realizations of the regional mean temperature.

For the change in regional mean temperature used as an input in $(6)$, we define $\bar{\Delta}_{S}^{(s, B)}(t)=$ $\beta_{1, S} C^{(s)}(t)$, which is, under the above model, the change in regional mean temperature from the preindustrial climate excluding changes in the seasonal cycle. For the changes in local mean temperature used as an input in the simulation (4), we assume that the local means are related to the regional means through a regional pattern scaling relationship; that is, the change in local mean from the preindustrial climate is taken to be proportional to the change in regional mean from preindustrial climate:

$$
\tilde{\mu}_{l}^{(s)}(t)-\tilde{\mu}_{l}^{(B)}=\lambda_{l}\left(\bar{\mu}_{S}^{(s)}(t)-\bar{\mu}_{S}^{(B)}\right) .
$$

This is the approach advocated by Castruccio et al. (2014) for grid cell-level mean emulation.

\section{S2 Choice of variable bandwidth for estimating $\delta_{l i}(\omega)$}

Here we describe the choice of weights, $w_{k, j, i}$, used in the estimates of $\delta_{l, i}^{(s)}\left(\omega_{j}\right)$ given by $(13)$. We let $w_{k, j, i}$ correspond to a variable-bandwidth quadratic kernel,

$$
w_{k, j, l} \propto \begin{cases}1-\left(\frac{k}{M_{j, i}+1}\right)^{2}, & k \in\left\{-M_{j, i}, \ldots, M_{j, i}\right\} \\ 0, & \text { otherwise }\end{cases}
$$

where $M_{j, i}$ controls the bandwidth at frequency $\omega_{j}$ for $i=0,1$, which we allow to vary as $M_{j, i}=$ $\left(m_{1, i}-m_{0, i}\right) h_{j}\left(p_{i}\right)+m_{0, i}$ where $m_{1, i}$ and $m_{0, i}$ are such that $0<m_{0, i} \leq m_{1, i}$ and

$$
h_{j}\left(p_{i}\right)= \begin{cases}\frac{1}{2}\left(1+\cos \left(\frac{2 \pi}{p_{i}}\left(\omega_{j}-\frac{p_{i}}{2}\right)\right)\right), & 0 \leq \omega_{j}<\frac{p_{i}}{2} \\ 1 & p_{i} \leq \omega_{j} \leq 1-p_{i} \\ \frac{1}{2}\left(1+\cos \left(\frac{2 \pi}{p_{i}}\left(\omega_{j}-\frac{p_{i}}{2}\right)\right)\right) & 1-\frac{p_{i}}{2}<\omega_{j}<1,\end{cases}
$$




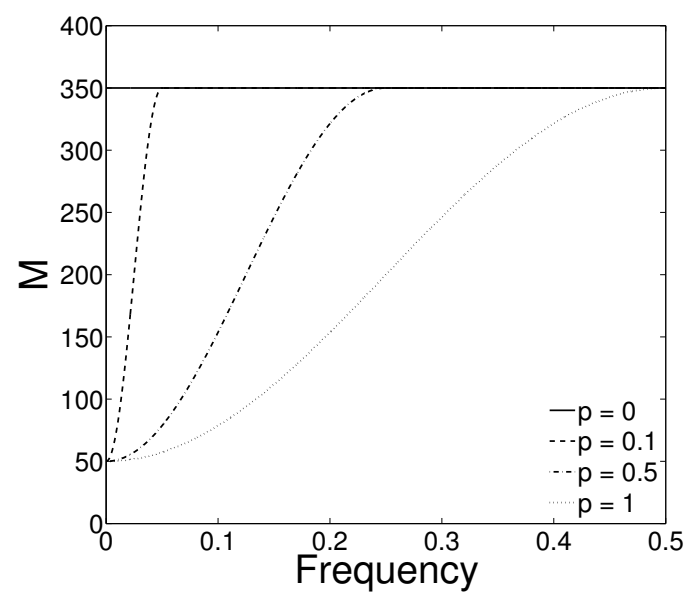

Figure S2: Examples of the function $M_{j, i}$ controlling the bandwidth of the kernel estimator as a function of the frequency being estimated. In each of these curves $m_{0, i}=50$ (controlling the minimum bandwidth) and $m_{1, i}=350$ (controlling the maximum bandwidth), and the value of $p_{i}$ (controlling the transition between the minimum and maximum bandwidths) is noted in the figure. The form of $M_{j, i}$ is such that the bandwidth of the kernel is smaller at low frequencies.

so $m_{1, i}$ controls the maximum bandwidth, $m_{0, i}$ the minimum bandwidth, and $p_{i}$ the frequency at which the bandwidths transition between $m_{0, i}$ and $m_{1, i}$ (see Figure S2). The reason we allow the bandwidths to decrease in frequency in this constrained way is that we have found that the log ratio of spectra in our ensemble are typically less smooth at very low frequencies. The specific form of $h$ is somewhat arbitrary, but appears to work well in practice.

To implement the estimator, we need to choose the parameters $m_{1, i}, m_{0, i}$, and $p_{i}$. This is done via leave-one-out cross validation; that is, for each region the parameters are chosen to minimize the sum of the cross-validation scores for each location,

$$
R_{C V}\left(m_{1, i}, m_{0, i}, p_{i}\right)=\sum_{j, l} \frac{\left(\hat{\delta}_{l i}\left(\omega_{j}\right)-\delta_{l i}^{*}\left(\omega_{j}\right)\right)^{2}}{\left(1-w_{0, j, i}\right)^{2}}
$$

where $\delta_{l i}^{*}$ is the maximizer of the approximate likelihood, (11), and $\hat{\delta}_{l i}$ is the smoothed version with weights $w_{k, j, l}$ defined above. These parameters vary by region, but typical values of $\left(m_{1, i}, m_{0, i}, p_{i}\right)$ when estimating $\delta_{l 0}$ and $\delta_{l 1}$ using all runs in our ensemble are around $(350,50,0.4)$ for $i=0$ and $(800,400,0.7)$ for $i=1$ (recall that the local periodograms in (11) are taken over ten-year blocks, so the raw estimates of $\delta_{l i}$ are calculated at 1,825 frequencies). Since the parameters vary regionally, there may be sudden changes in estimates of the changes in spectra near boundaries of the region (see Figure 6, top right). One could consider a post-hoc spatial smoothing of either the bandwidths or the changes in spectra to avoid this, but this is not explored in this paper. 


\section{S3 Standard errors for estimates of $\delta_{l i}(\omega)$}

Under (11), the Fisher information for $\left(\delta_{l 0}, \delta_{l 1}, \tilde{a}^{(B)}\right)$ is a block diagonal matrix with blocks for each frequency, $\omega_{j}$, approximately (under standard approximations for periodograms) equal to

$$
\mathcal{I}\left(\omega_{j}\right)=\left[\begin{array}{ccc}
\sum_{s b}\left(\bar{\Delta}_{b}^{(s, B)}\right)^{2} R_{s}^{\prime} & \sum_{s b}\left(\partial_{t} \bar{\Delta}_{b}^{(s, B)}\right) \bar{\Delta}_{b}^{(s, B)} R_{s}^{\prime} & \sum_{s b} \frac{\bar{\Delta}_{b}^{(s, B)} R_{s}^{\prime}}{\tilde{a}^{(B)}\left(\omega_{j}\right)} \\
& \sum_{s b}\left(\partial_{t} \bar{\Delta}_{b}^{(s, B)}\right)^{2} R_{s}^{\prime} & \sum_{s b} \frac{\partial_{t} \bar{\Delta}_{b}^{(s, B)} R_{s}^{\prime}}{a^{(B)}\left(\omega_{j}\right)} \\
& & \sum_{s b} \frac{R_{s}^{\prime}+M}{\tilde{a}^{(B)}\left(\omega_{j}\right)^{2}}
\end{array}\right]
$$

where $R_{s}^{\prime}=R_{s}-1$. We calculate standard errors under the usual approximation that the variance of the maximum likelihood estimate is the inverse information matrix; such an approximation can be justified by, for example, considering either the number of independent GCM runs or the number of time blocks to be large. The resulting covariance matrix for $\delta_{l 0}^{*}\left(\omega_{j}\right)$ and $\delta_{l 1}^{*}\left(\omega_{j}\right)$, the maximum likelihood estimators for the $\delta$ functions, is constant in frequency. Write

$$
\begin{aligned}
\operatorname{Var}\left(\begin{array}{c}
\delta_{l 0}^{*}\left(\omega_{j}\right) \\
\delta_{l 1}^{*}\left(\omega_{j}\right)
\end{array}\right) & \approx\left[\begin{array}{ll}
\mathcal{I}\left(\omega_{j}\right)_{1,1}^{-1} & \mathcal{I}\left(\omega_{j}\right)_{1,2}^{-1} \\
& \mathcal{I}\left(\omega_{j}\right)_{2,2}^{-1}
\end{array}\right] \\
& \equiv\left[\begin{array}{ll}
V_{\delta_{l 0}, \delta_{l 0}} & V_{\delta_{l 0}, \delta_{l 1}} \\
& V_{\delta_{l 1}, \delta_{l 1}}
\end{array}\right]
\end{aligned}
$$

Then the variance of the smoothed estimates, $\hat{\delta}$, defined in (13), at frequencies not close to 0 or $\pi$ is

$$
\begin{aligned}
\operatorname{Var}\left(\begin{array}{c}
\hat{\delta}_{l 0}\left(\omega_{j}\right) \\
\hat{\delta}_{l 1}\left(\omega_{j}\right)
\end{array}\right) & \equiv\left[\begin{array}{ll}
V_{\hat{\delta}_{l 0}, \hat{\delta}_{l 0}} & V_{\hat{\delta}_{l 0}, \hat{\delta}_{l 1}} \\
& V_{\hat{\delta}_{l 1}, \hat{\delta}_{l 1}}
\end{array}\right] \\
& =\left[\begin{array}{cc}
V_{\delta_{l 0}, \delta_{l 0}} \sum_{k} w_{k, j, 0}^{2} & V_{\delta_{l 0}, \delta_{l 1}} \sum_{k} w_{k, j, 0} w_{k, j, 1} \\
& V_{\delta_{l 1}, \delta_{l 1}} \sum_{k} w_{k, j, 1}^{2}
\end{array}\right]
\end{aligned}
$$

When 0 or $\pi$ is within the local bandwidth for the frequency of interest, we make the correction to the above to account for the fact that $\delta_{l i}^{*}\left(\omega_{j}\right)$ is periodic and even (here omitted for simplicity). The variance of $\log \hat{\rho}_{l}^{(s, B)}(t, \omega)$, the smoothed estimate of log ratio of spectra, is then approximately

$$
\begin{aligned}
\operatorname{Var} \log \hat{\rho}_{l}^{(s, B)}(t, \omega) \approx\left(\bar{\Delta}_{S}^{(s, B)}(t)\right)^{2} V_{\hat{\delta}_{l 0}, \hat{\delta}_{l 0}}+\left(\partial_{t} \bar{\Delta}_{S}^{(s, B)}(t)\right)^{2} V_{\hat{\delta}_{l 1}, \hat{\delta}_{l 1}} \\
+2 \bar{\Delta}_{S}^{(s, B)}(t) \partial_{t} \bar{\Delta}_{S}^{(s, B)}(t) V_{\hat{\delta}_{l 0}, \hat{\delta}_{l 1}}
\end{aligned}
$$




\section{S4 Computing simulations}

Computing (4) efficiently requires the ability to quickly compute the products $C_{N_{T}}\left(\sqrt{\hat{\rho}^{(s, B)}}\right) x$ and $C_{N_{T}}^{-1}\left(\sqrt{\hat{\rho}^{(0, B)}}\right) x$ for a vector $x$. The matrix-vector products may be written as

$$
\begin{aligned}
& \left(C_{N_{T}}\left(\sqrt{\hat{\rho}^{(s, B)}}\right) x\right)_{t}=\sqrt{\frac{2 \pi}{N_{T}}} \sum_{j=0}^{N_{T}-1} x_{j} e^{\frac{1}{2}\left[\bar{\Delta}_{t}^{(s, B)} \hat{\delta}_{l 0}\left(\omega_{j}\right)+\partial_{t} \bar{\Delta}_{t}^{(s, B)} \hat{\delta} l 1\left(\omega_{j}\right)\right]} e^{i \omega_{j} t} \\
& =\sqrt{\frac{2 \pi}{N_{T}}} \sum_{j=0}^{N_{T}-1} x_{j} \sum_{p=0}^{\infty} \frac{\left\{\frac{1}{2}\left[\bar{\Delta}_{t}^{(s, B)} \hat{\delta}_{l 0}\left(\omega_{j}\right)+\partial_{t} \bar{\Delta}_{t}^{(s, B)} \hat{\delta}_{l 1}\left(\omega_{j}\right)\right]\right\}^{p}}{p !} e^{i \omega_{j} t} \\
& \approx \sqrt{\frac{2 \pi}{N_{T}}} \sum_{p=0}^{P-1} \sum_{m=0}^{p} \frac{\left(\bar{\Delta}_{t}^{(s, B)}\right)^{p-m}\left(\partial_{t} \bar{\Delta}_{t}^{(s, B)}\right)^{m}}{2^{p} m !(p-m) !} \sum_{j=0}^{N_{T}-1} \hat{\delta}_{l 0}\left(\omega_{j}\right)^{p-m} \hat{\delta}_{l 1}\left(\omega_{j}\right)^{m} x_{j} e^{i \omega_{j} t}
\end{aligned}
$$

where the approximation just truncates the Taylor series at $P-1$, then uses the binomial expansion and changes the order of summation. This approximation is the weighted sum of $P(P+1) / 2$ inverse discrete Fourier transforms; if $P$ can be taken to be only modestly large so that the Taylor approximation is accurate, this sum can be computed efficiently. In our setting, the changes in variability projected by the GCM are small and $P$ need not be very large in order for the approximation to be quite good; we have found that taking $P=10$ is more than enough to give accurate approximations given the magnitude of the estimated changes in variability.

To compute the matrix-inverse vector products, we have found, as in Guinness and Stein (2013), that iterative methods work well. In order to work well, these require the ability to compute forward multiplication quickly, which we have just described, as well as a good preconditioner; since the historical time series is only mildly nonstationary, we have found that the Fourier transform scaled by the square root of the average of $\rho^{(0, B)}(t, \omega)$ over time works well as a preconditioner. 


\section{S5 Additional supplementary figures}
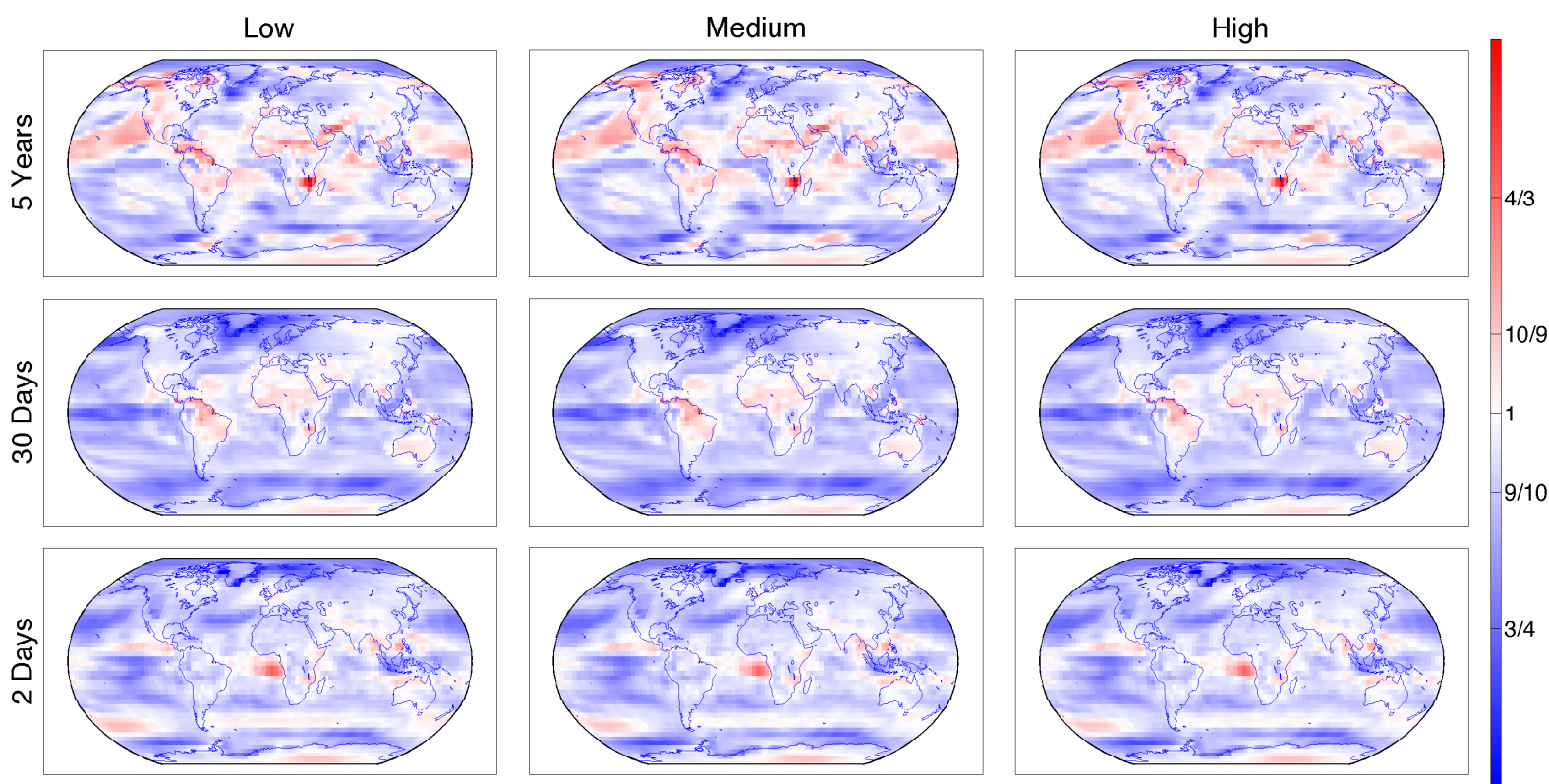

Figure S3: Estimates of changes in marginal spectra, at three frequencies, at the year corresponding to that with the same regional mean temperature as in the low scenario in model year 2100 (i.e., left, $\rho_{l}^{(L, B)}(t, \omega)$ at year 2100; middle, $\rho_{l}^{(M, B)}(t, \omega)$ at years ranging from 2050-2056 depending on region; right, $\rho_{l}^{(H, B)}(t, \omega)$ at years ranging from 2037-2044 depending on region). Red indicates an increase in variability and blue a decrease in variability. The estimated differences between scenarios are small because the effect of the rate of change of warming on the estimated changes in variability are smaller than the effect of the overall regional mean change in temperature. A direct comparison of the estimates between the high and low scenario is shown in Figure 3 of the main text. 


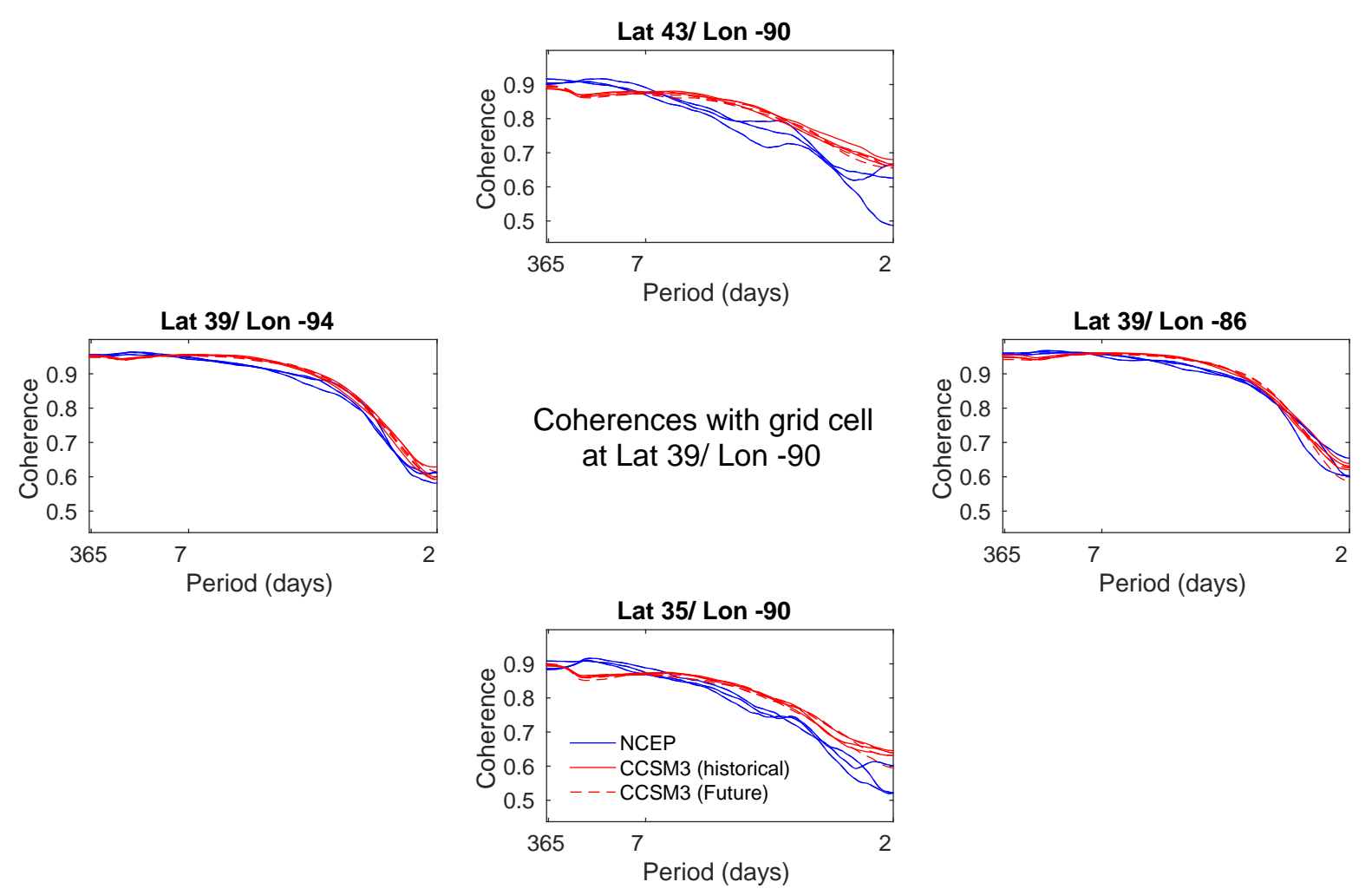

Figure S4: Estimates of coherence spectra between temperatures in the Midwestern grid cell discussed in Section 5.1 and its four neighboring grid cells; the coordinates corresponding to each neighbor are indicated (and figures are positioned geographically). Estimates are shown for the ten-year blocks 1980-1989, 1990-1999, and 2000-2009 as well as the corresponding time 90 years in the future under the high scenario (i.e., 2070-2079, 2080-2089, and 2090-2099). Estimates in red correspond to temperatures from CCSM3 under historical forcing (solid) and in the high scenario future (dashed). Those in blue correspond to temperatures from NCEP CFSR and the simulation (the coherences do not change in the simulation). The time series were processed to remove means and (marginal) seasonal variability as described in the main text. Marginal spectra, co-, and quadrature spectra were estimated by averaging the raw estimates for each run (for the CCSM3 runs, where we have multiple runs) and then smoothing each component using a quadratic kernel; since we cannot average over multiple runs of reanalysis, the estimates for reanalysis were smoothed using a slightly wider bandwidth. While the coherence spectra are arguably similar in the East-West direction, CCSM3 has temperatures that are more coherent than the reanalysis's in the North-South direction. Coherences do not change much in the future GCM run, which suggests that our approach for the simulations may be adequate. 


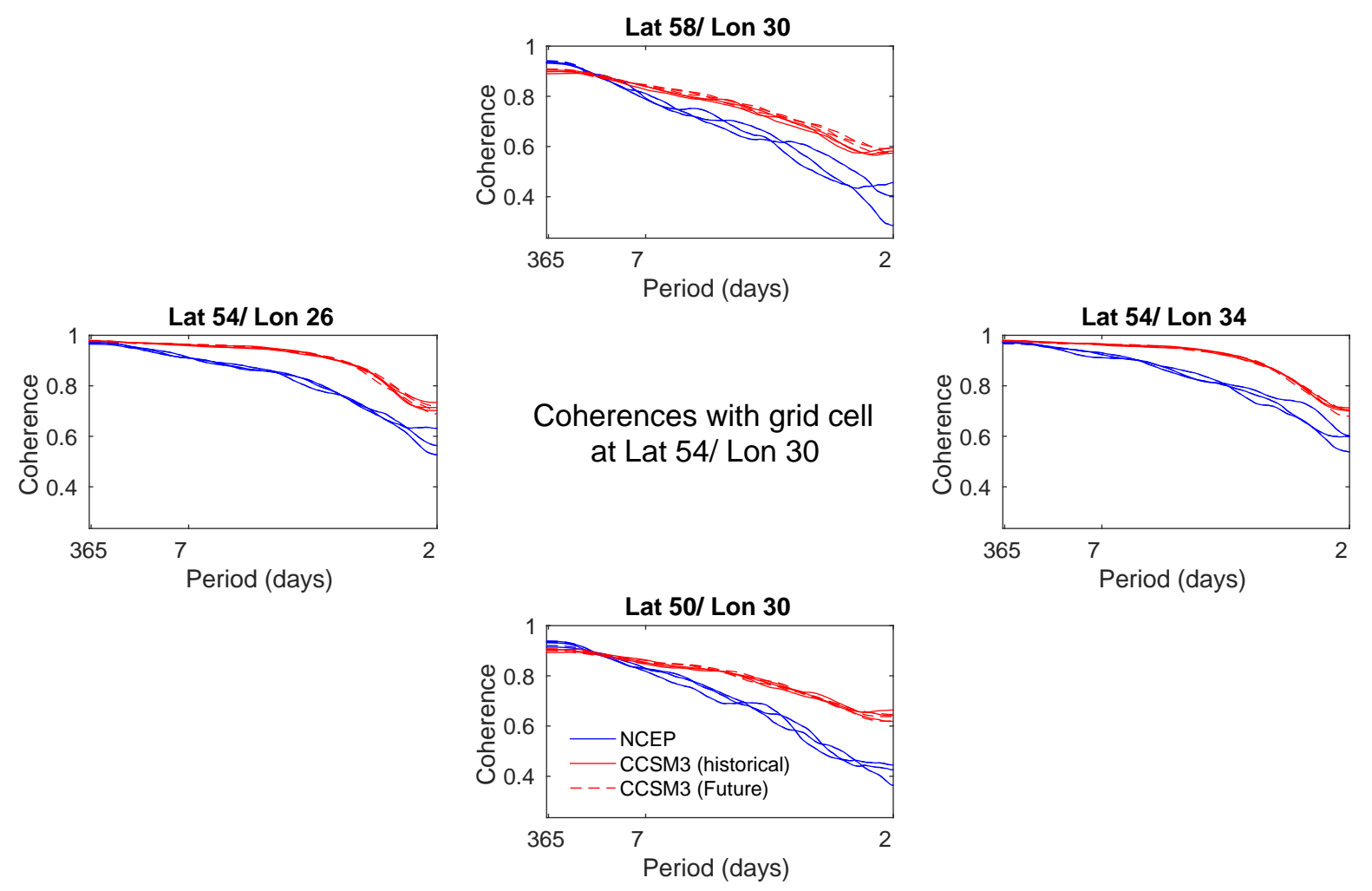

Figure S5: The same as Figure S4 but for a central grid cell in Northern Europe. Temperatures are more coherent in CCSM3 than in the reanalysis. 

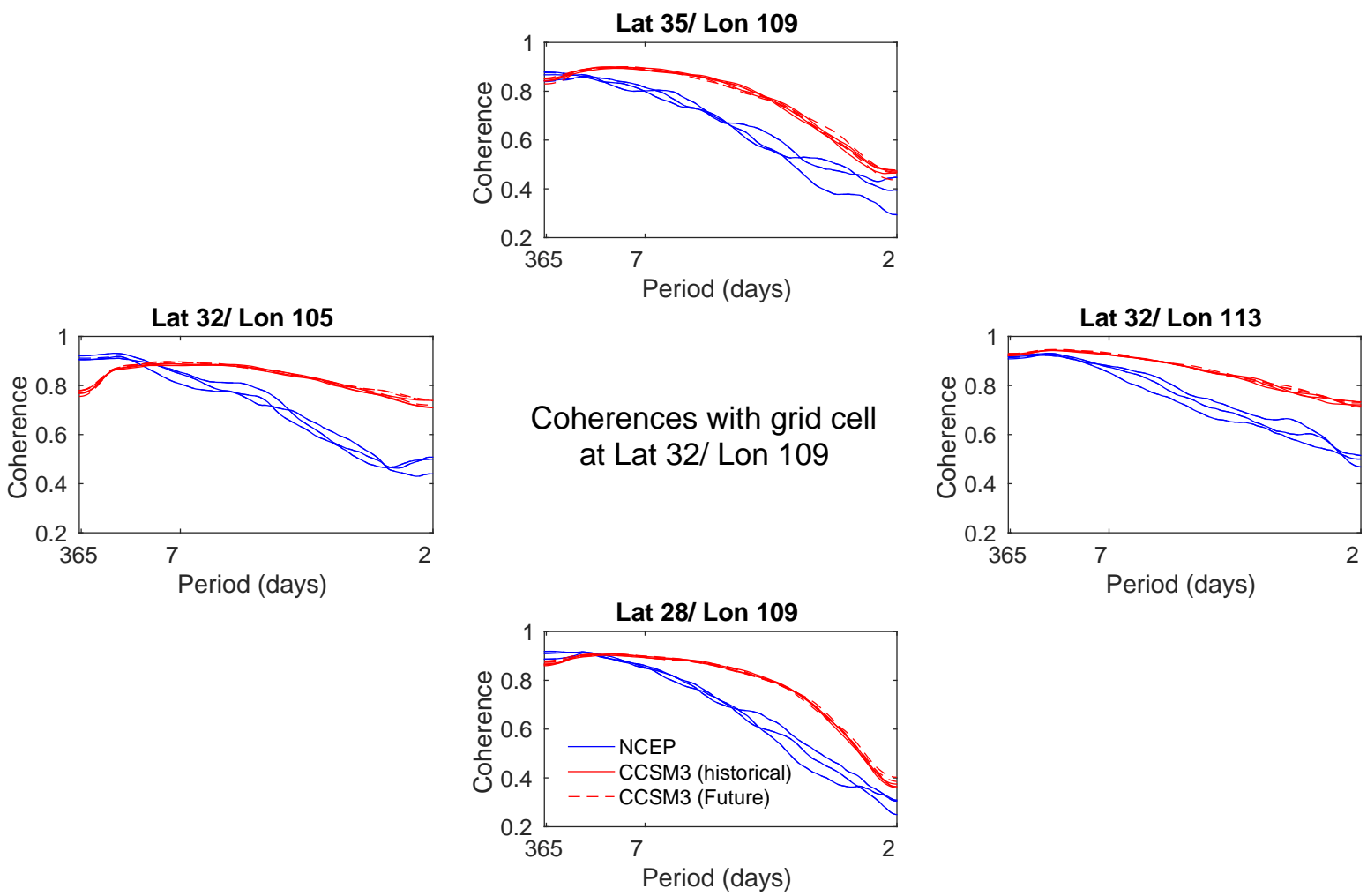

Figure S6: The same as Figure S4 but for a central grid cell in East Asia. Temperatures are more coherent in CCSM3 than in the reanalysis. 


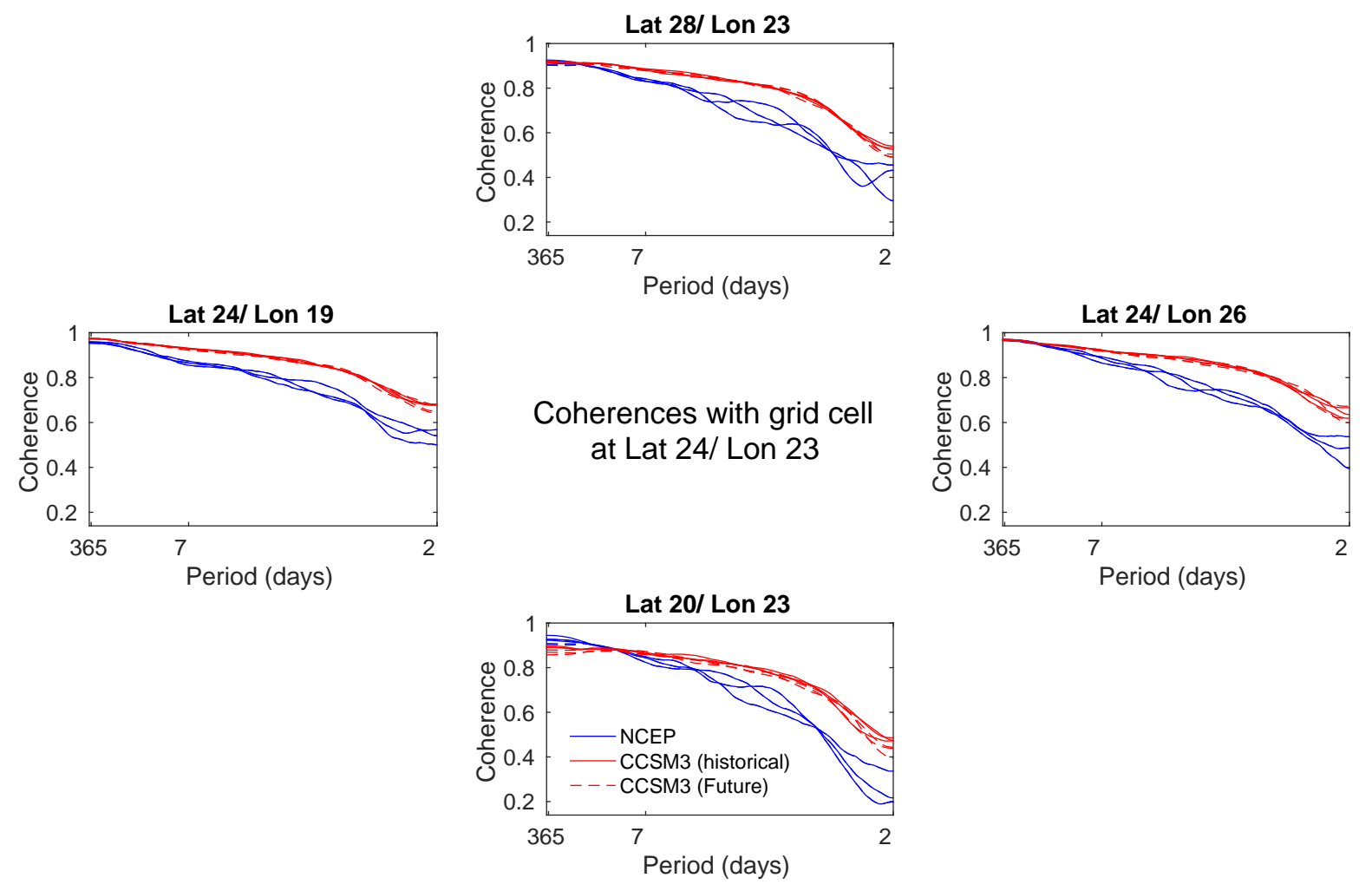

Figure S7: The same as Figure S4 but for a central grid cell in the Sahara. Temperatures are more coherent in CCSM3 than in reanalysis. 


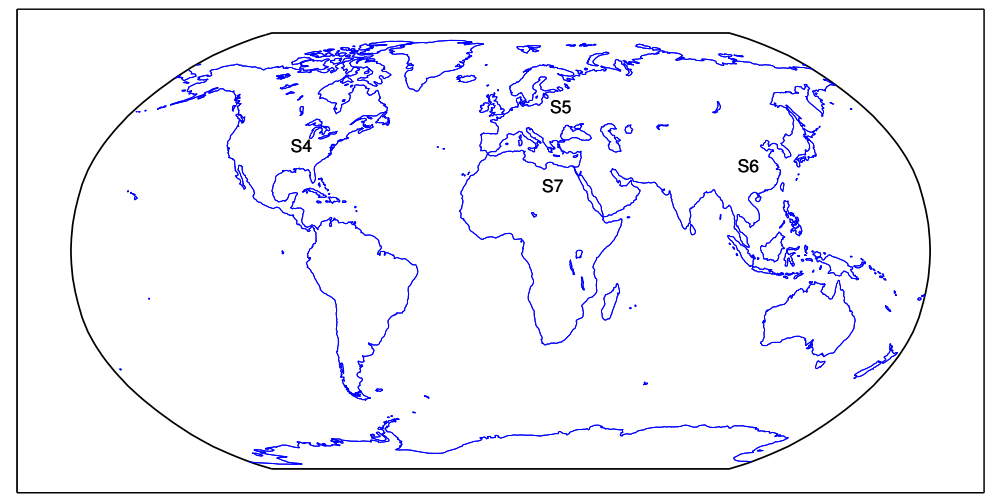

Figure S8: The locations of the central grid cells corresponding to the coherency plots in Figures S4 - S7 (labeled by figure number). The grid cell for Figure S4 corresponds to the one discussed in Section 5.1 of the main text.

\section{S6 Description of animated simulation}

An animation of a full global simulation using the methods described in the main text is given in the supplementary file "Simulation.mp4". On the top row, the animation shows raw temperatures from NCEP CFSR from the years 1979-2009 (left), our resulting simulation under the high scenario for the years 2069-2099 (middle), and the difference between the simulation and raw reanalysis (right). The simulation has an increased mean (stronger in the higher latitudes) and changes in variability of the nature shown in the main text and supplementary figures. The animation demonstrates that the simulation retains many of the space-time features of the observational record. On the bottom row of the animation, we show output from one run of CCSM3 under the same years and scenario. (Since the CCSM3 runs do not have leap days, these days are left blank in the animation to keep synchronous with the observations and simulation in the top row.) The CCSM3 temperatures appear lower than the corresponding reanalysis or simulation. Differences in variability between the GCM and reanalysis/simulation are more difficult to see by eye; these are explored in Figures 4 and S4 - S7.

\section{References}

Stefano Castruccio, David J McInerney, Michael L Stein, Feifei Liu Crouch, Robert L Jacob, and Elisabeth J Moyer. Statistical emulation of climate model projections based on precomputed gcm runs. Journal of Climate, 27(5):1829-1844, 2014.

Joseph Guinness and Michael L Stein. Transformation to approximate independence for locally stationary gaussian processes. Journal of Time Series Analysis, 34(5):574-590, 2013. 